\title{
The safety and efficacy of amrubicin in the treatment of previously untreated extensive-disease small-cell lung cancer: a meta-analysis
}

This article was published in the following Dove Press journal:

OncoTargets and Therapy

\author{
Ji-Feng $W u^{\prime}$ \\ Jian-Jun Zhou' \\ Xin-Ai Li ${ }^{1}$ \\ Li-Hui Hu' \\ Meng-Li Wen ${ }^{2}$ \\ 'Department of Respiratory Medicine, \\ Jiangxi Province Hospital of Integrated \\ Chinese \& Western Medicine, Nanchang, \\ Jiangxi, People's Republic of China; \\ ${ }^{2}$ Department of General surgery, The \\ Fourth Affiliated Hospital of Nanchang \\ University, Nanchang, Jiangxi, People's \\ Republic of China
}

Correspondence: Ji-Feng Wu Department of Respiratory Medicine, Jiangxi Province Hospital of Integrated

Chinese \& Western Medicine, No.90 Bayi

Avenue, Nanchang, Jiangxi 330003,

People's Republic of China

Tel +86 I0I 8970025485

Email wujifeng_918@I63.com
Background: Extensive-disease small-cell lung cancer (ED-SCLC) has been known to be rapid progression and relapse, despite highly sensitive to chemotherapy. Amrubicin (AMR), a third-generation synthetic anthracycline, was accepted as a feasible alternative compared with the standard first-line chemotherapy for previously untreated ED-SCLC. While, the efficacies of these amrubicin-based regimens are unsatisfactory.

Aim: Our meta-analysis was performed to assess the efficacy and toxicity of first-line therapy comparing AMR and chemotherapy in patients with ED-SCLC.

Methods: Electronic databases were searched for eligible trials updated on November 2018. Randomized-controlled trials assessing the efficacy and safety of AMR in ED-SCLC were included, of which the interested results were objective response rate (ORR), progressionfree survival (PFS), overall survival (OS), and adverse events (AEs).

Results: A total of 6 randomized controlled trials were included in this analysis. There are no significant differences in $\mathrm{OS}(\mathrm{OR}=1.03,95 \% \mathrm{CI}=0.66-1.60, P=0.91)$, PFS (OR=1.2, 95\% $\mathrm{CI}=10.77-1.88, P=0.41)$ or $\mathrm{ORR}(\mathrm{OR}=1.31,95 \% \mathrm{CI}=0.90-1.92, P=0.16)$ with $\mathrm{AMR}$ $(\mathrm{OR}=0.90,95 \% \mathrm{CI}=0.76-1.05, P=0.17)$. The most common treatment-related AEs in the AMR group are leukopenia $(\mathrm{OR}=3.13,95 \% \mathrm{CI}=1.22-7.99, P=0.02)$ and neutropenia $(\mathrm{OR}=3.25,95 \% \mathrm{CI}=1.38-7.65, P=0.007)$. Fatigue, anemia, nausea, vomiting, diarrhea the difference between the two groups had no statistical significance.

Conclusion: The results of our analysis indicated that AMR therapy demonstrated noninferiority to the standard first-line chemotherapy for previously untreated ED-SCLC. Whether it can be accepted as an alternative regimen to the standard first-line chemotherapy is still warranted.

Keywords: small-cell lung cancer, extensive-disease, amrubicin, meta-analysis

\section{Introduction}

Lung cancer is the leading cause of cancer-associated death in the world, ${ }^{1}$ and small-cell lung cancer (SCLC) accounts for approximately $20 \%$ cases. $^{2}$ More than half of the cases are diagnosed with extensive-disease (ED) SCLC, which is characterized by rapid progression. ${ }^{3}$ Despite being highly initial response rates to chemotherapy, SCLC has developed into drug resistance with poor survival. ${ }^{3}$ Thus, there is a need for development of new and effective therapies for ED-SCLC.

Standard drugs to treat SCLC include cyclophosphamide, etoposide, doxorubicin, vincristine, methotrexate, cisplatin, and carboplatin. The combination chemotherapy using a platinum-based drug plus etoposide has been accepted as the standard treatment 
for first-line treatment for ES-SCLC. ${ }^{4}$ Moreover, both irinotecan plus cisplatin (IP) and etoposide plus cisplatin (EP) have the similar efficacy and are considered as a standard EDSCLC treatment in Japan. ${ }^{5,6}$ However, significant symptomatic non-hematological toxicities are associated with the administration of cisplatin and include gastrointestinal, neural and renal function failure, and electrolyte disturbance. Despite the development in treatment strategies of SCLC with targeted agents and newer chemotherapies, ${ }^{7-9}$ the results for SCLC patients have not been significantly developed.

Amrubicin, a completely synthetic anthracycline derivative, is converted to an active metabolite amrubicinol in the liver and a potent topoisomerase II inhibitor. ${ }^{10}$ Amrubicin as single-agent provided response rates of $75.8 \%$, with a median survival time of 11.7 months, while when combine therapy with cisplatin yielded a high response rates of $87.8 \%$ and median survival durations of 13.6 months for previously untreated ED-SCLC. ${ }^{11,12}$ These promising results support examining amrubicin as a viable SCLC treatment.

However, previous studies have reported controversial and sometimes conflicting results because of their toxicity or limited efficacy that are rarely found in previously untreated patients with ED-SCLC. The objective of this meta-analysis is to identify the efficacy and toxicity of AMR as a promising treatment option for ED-SCLC.

\section{Methods and materials}

\section{Retrieval strategy}

Published articles about the efficacy and safety of AMR as a promising treatment option for ED-SCLC up to November 2018 were retrieved. The searchable databases included PubMed, EMBASE, and Cochrane library, and the following keywords were used: "small-cell lung cancer" AND "extensive-disease" AND "amrubicin", and no limitation was used during the literature search (("smallcell lung cancer" OR "small-cell lung carcinoma" OR "SCLC") AND ("extensive-disease" OR "ED-SCLC") AND (1st-line OR “first line" OR "previously untreated") AND (amrubicin OR AMR OR Calsed OR SM-5887)). The references of eligible studies that dealt with the topic of interest were also manually searched to identify additional relevant publications. The study was designed according to PRISMA Checklist.

\section{Eligibility criteria}

Articles that were related to the following inclusion criteria were included in this analysis: (1) the studies are designed as random control trials (RCTs); (2) trials focused on comparing AMR and chemotherapy; (3) the two groups provide complete data were treated patients with previously untreated ED-SCLC; (4) the results of interested were efficacy and toxicity, and HRs with corresponding 95\% confidence interval $(95 \% \mathrm{CI})$ were provided; (5) the full texts were only included. Studies with complete information would be included from overlapped or duplicated data in multiple reports.

\section{Quality assessment}

Two investigators separately assessed the quality of the retrieved studies. The risk of bias items (ROBI) recommended by The Cochrane Handbook for Systematic Reviews of Interventions was used.

\section{Data extraction}

Two authors extracted the relevant data from individual studies separately, and differences were settled through discussion. The main categories were based on the following parameters from the eligible studies: the names of authors, publication year, treatment regimen, sample size, mean age, and the outcomes of interest. We extracted the corresponding variables adjusted and risk estimates of mortality with $95 \%$ CIs.

\section{Risk of bias}

After assessing the online databases, only 6 RCTs were included. This is not enough to conduct Begg funnel plot to evaluate publication bias.

\section{Statistical analysis}

Review Manager version 5.3 software (Revman; The Cochrane collaboration Oxford, United Kingdom) was used to perform further statistical analyses. A sensitivity analysis was also performed to examine the impact on the overall results, depending on the heterogeneity across the included studies. To assess the heterogeneity of study trial and determine the model for analysis, $\mathrm{I}^{2}$ statistic and Chisquared test were conducted. ${ }^{14}$ Fixed-effect model was used if the assessment of heterogeneity was insignificant $\left(\mathrm{I}^{2} \leq 50 \%\right)$. If the source of heterogeneity was not insignificant $\left(\mathrm{I}^{2}>50 \%\right)$ uncertain, we used the random-effect model for further analysis. ${ }^{15}$ A $P$-value less than 0.05 was identified as statistically significant difference. Forest plots indicated the findings of our meta-analysis. 


\section{Results}

\section{Overview of literature search and study characteristics}

Totally, 371 articles were identified initially. Based on the criteria described in the methods, 365 articles were excluded due to the lack of outcomes of 2 approaches. Therefore, a final total of 6 RCTs $^{6,15-19}$ were assessed for eligibility in the meta-analysis (Figure 1). All included studies in this study were based on moderate to high quality evidence. Table 1 provides a brief description of these 6 studies.

\section{Clinical and methodological heterogeneity Pooled analysis of PFS comparing AMR versus chemotherapy}

Pooling the PFS from studies showed that no benefit was found between $\mathrm{AMR}$ and chemotherapy $(\mathrm{OR}=1.2,95 \%$ $\mathrm{CI}=10.77-1.88, P=0.41)$, and the data are shown in Figure 2.

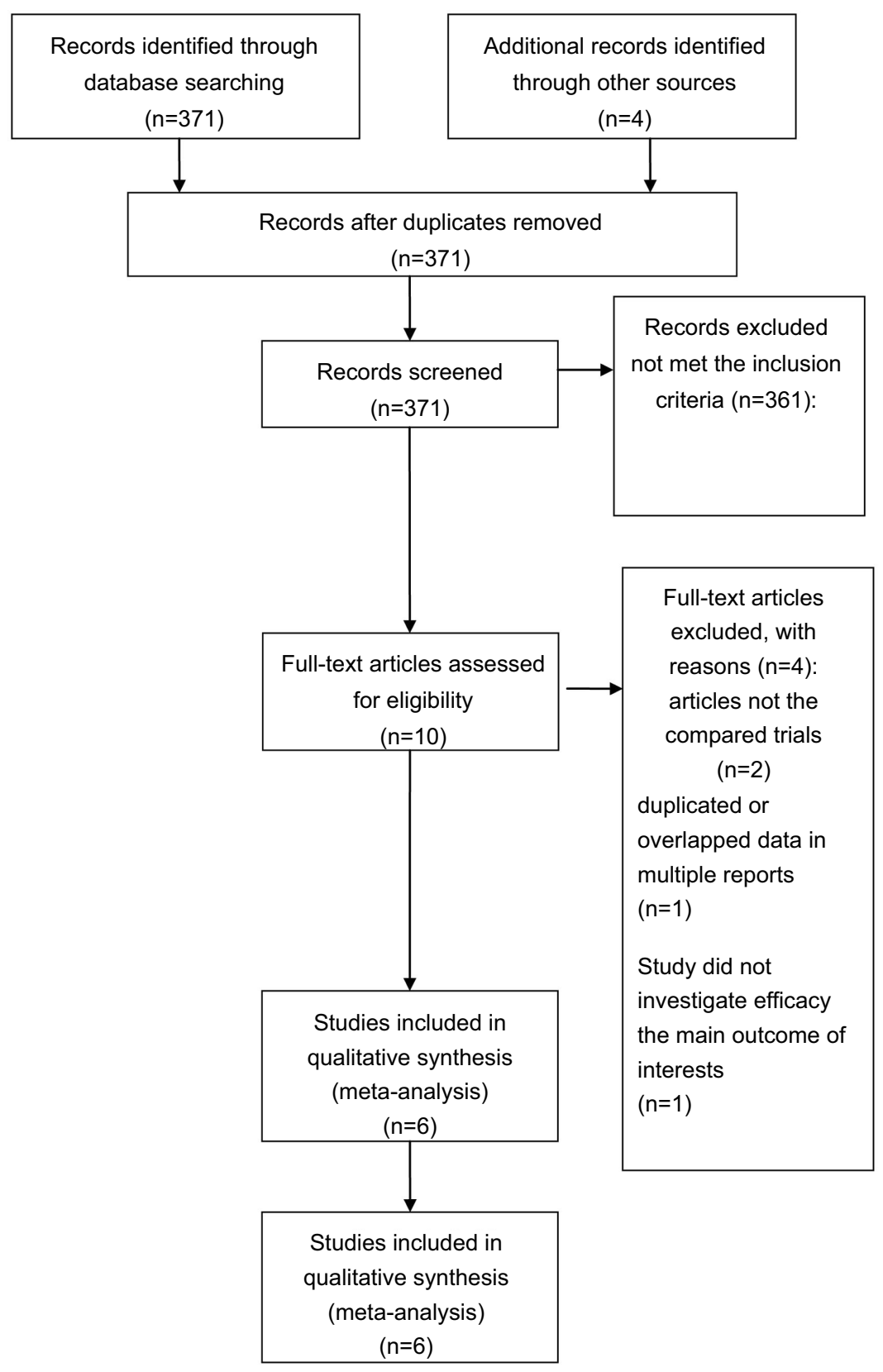

Figure I PRISMA flow chart of selection process to identify studies eligible for pooling. 


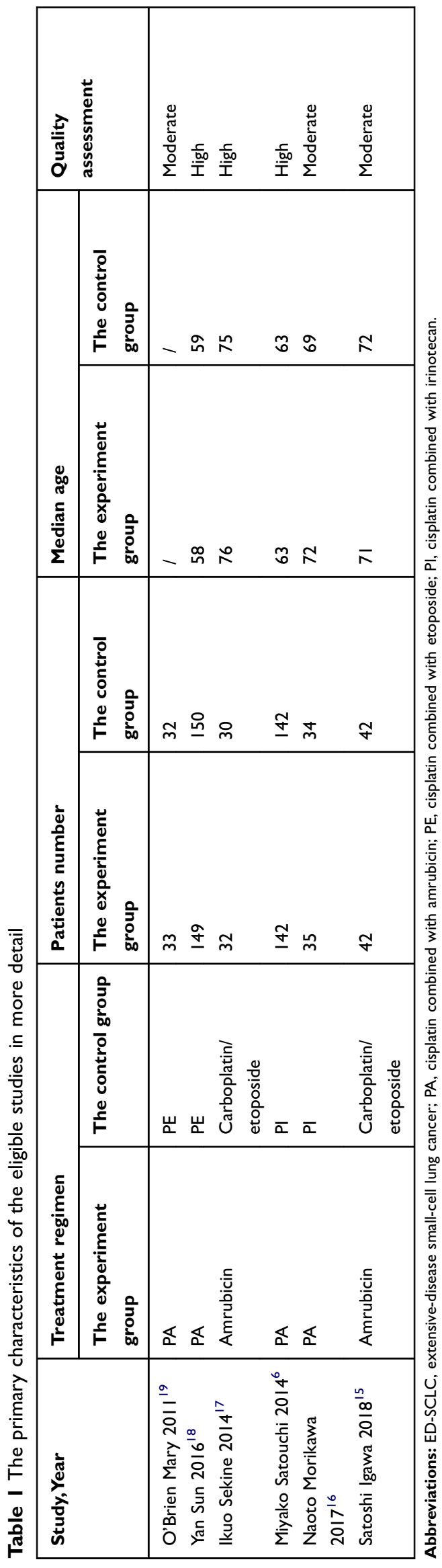

\section{Pooled analysis of OS comparing AMR versus chemotherapy}

Five trials reported the OS data. As displayed in Figure 3, pooled estimates of effect sizes showed no significant statistical difference of OS when comparing the two groups $(\mathrm{OR}=1.03,95 \% \mathrm{CI}=0.66-1.60, P=0.91)$.

\section{Pooled analysis of ORR comparing AMR versus chemotherapy}

Systematic evaluations of ORR are shown in Figure 4. The pooled results showed that there was no remarkable difference when comparing the two groups $(\mathrm{OR}=1.31,95 \%$ $\mathrm{CI}=0.90-1.92, P=0.16$ ).

\section{Pooled analysis of SAEs comparing AMR versus chemotherapy}

We define the grade $3 / 4$ toxicities as severe AEs. In the analysis, fatigue, anemia, nausea, vomiting, and diarrhea were included, and the data are shown in Figures 5-9. While, all above data did not reach a statistically significant level $(P>0.05)$. Moreover, the most common treatmentrelated adverse events in the AMR group are leukopenia (Figure 10) $(\mathrm{OR}=3.13,95 \% \mathrm{CI}=1.22-7.99, P=0.02)$ and neutropenia (Figure 11) $(\mathrm{OR}=3.25,95 \% \mathrm{CI}=1.38-7.65, P=0.007)$.

\section{Discussion}

SCLC represents approximately $15-20 \%$ of all lung cancers, ${ }^{4}$ and more than half of the cases are diagnosed with extensive-stage (ES) SCLC. ${ }^{3}$

ES-SCLC is chemosensitive due to the rapidly proliferating tumor. The standard treatment is systemic chemotherapy alone, which leads to tumor shrinkage and symptom relief in the majority of patients; however, the rapid progression of clinical drug resistance has resulted in poor prognosis. ${ }^{20}$ Thus, there is a need for new and effective therapy for ES-SCLC.

Recently, the Japanese Clinical Oncology group (JCOg) has reported the non-inferiority of amrubicin/cisplatin when compared to the irinotecan/cisplatin for previously untreated ED-SCLC. ${ }^{6}$ While, Satouchi ${ }^{15}$ did not achieve efficacy benefit with AP as standard first-line therapy for ED-SCLC.

In this meta-analysis, we found that non-inferiority but not superiority of AMR therapy to the control therapy. In other words, the AMR regimen did not achieve any efficacy benefit for chemo-naive patients with ES-SCLC. The results seen here do not underrate the efficacy of AMR in SCLC and perhaps stress the particular value of AMR as second- or third-line treatment in this setting. Although cisplatin plus amrubicin 


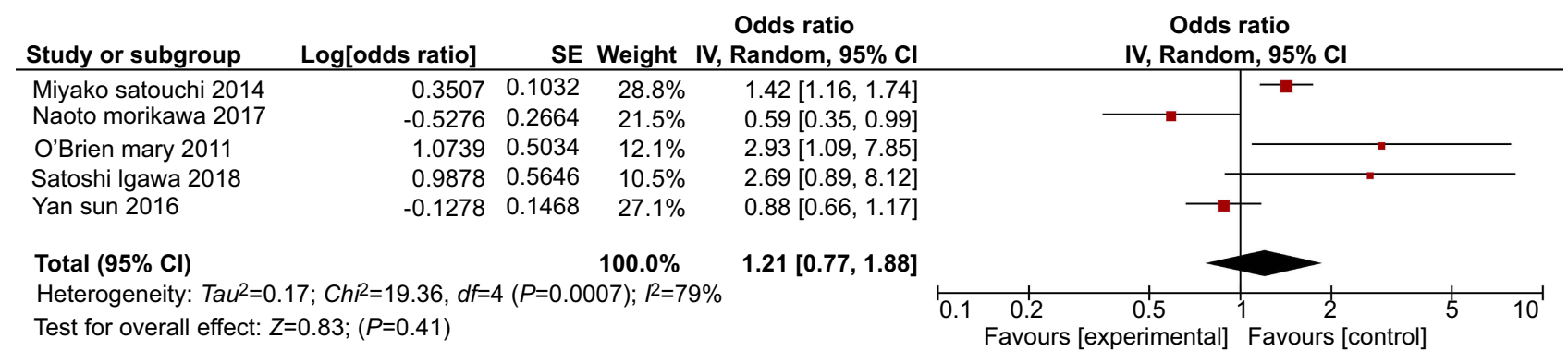

Figure 2 Pooled analysis of PFS comparing AMR versus chemotherapy.

Abbreviations: PFS, progression free survival; AMR, amrubicin.

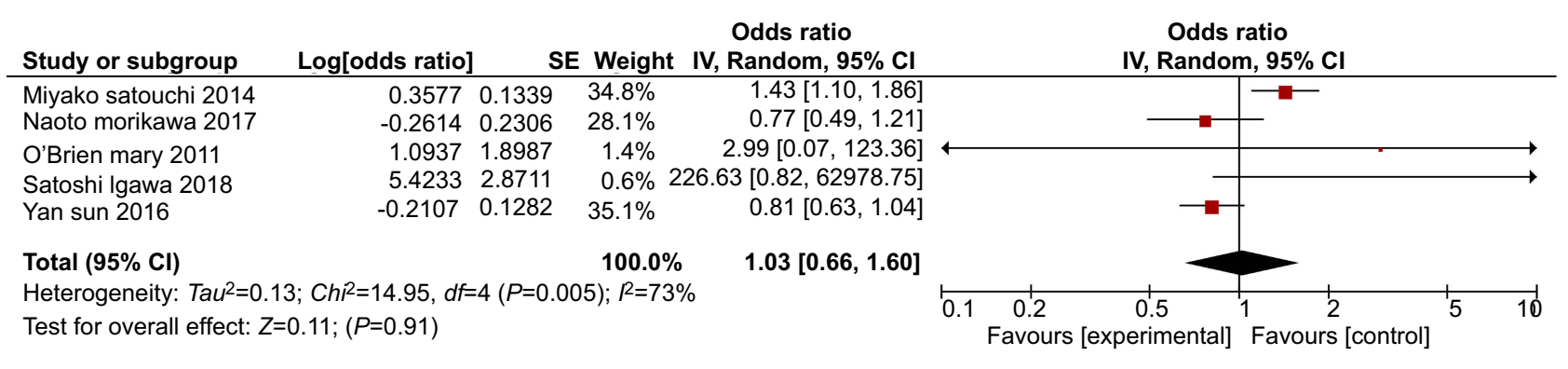

Figure 3 Pooled analysis of OS comparing AMR versus chemotherapy.

Abbreviations: OR, overall survival; AMR, amrubicin.

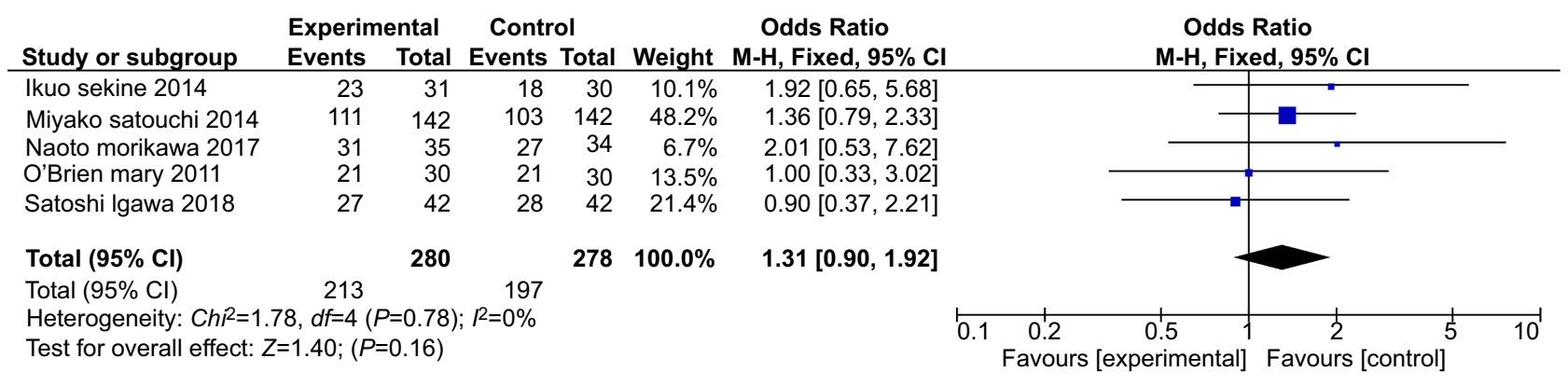

Figure 4 Pooled analysis of ORR comparing AMR versus chemotherapy. Abbreviations: ORR, objective response rate; $A M R$, amrubicin.

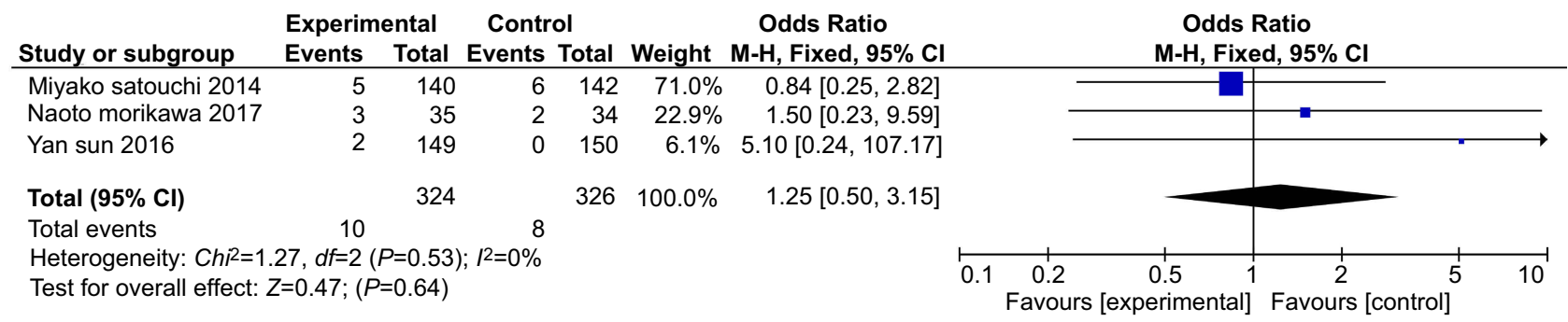

Figure 5 Pooled analysis of fatigue comparing AMR versus chemotherapy.

Abbreviation: AMR, amrubicin.

did not achieve benefit than other cisplatin-based therapy, ${ }^{6,18}$ the results of the Morikawas ${ }^{16}$ study and their previous trials $^{21,22}$ reported that the CBDCA-based therapy might be superior than the CDDP-based therapy with amrubicin. Moreover, as the sample size of some studies were too small, these results have low statistical power. 


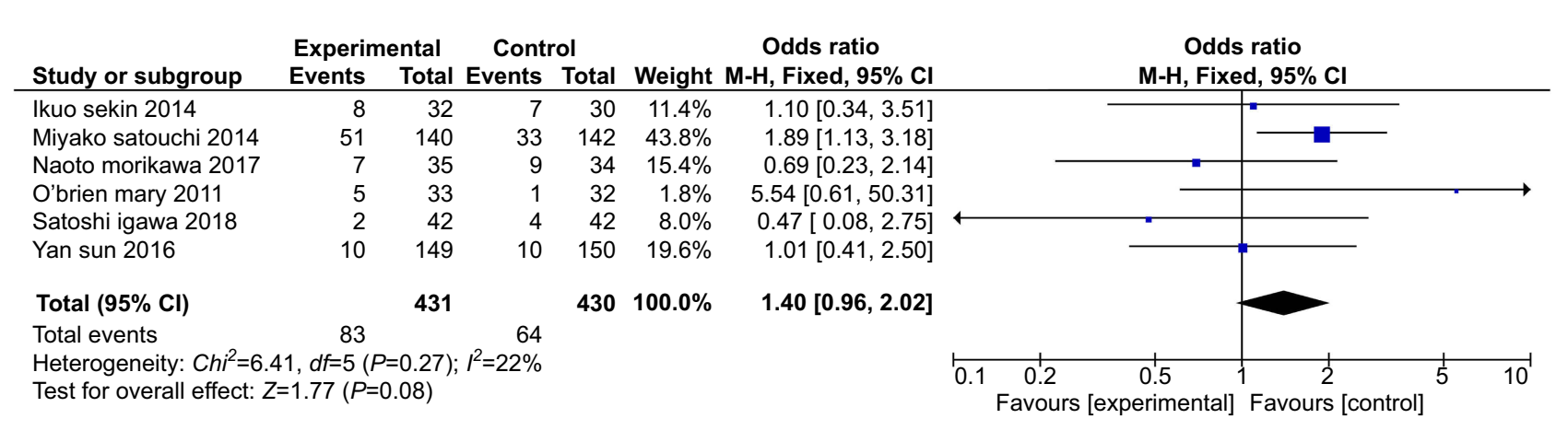

Figure 6 Pooled analysis of anemia comparing AMR versus chemotherapy. Abbreviations: PFS, progression free survival; AMR, amrubicin.

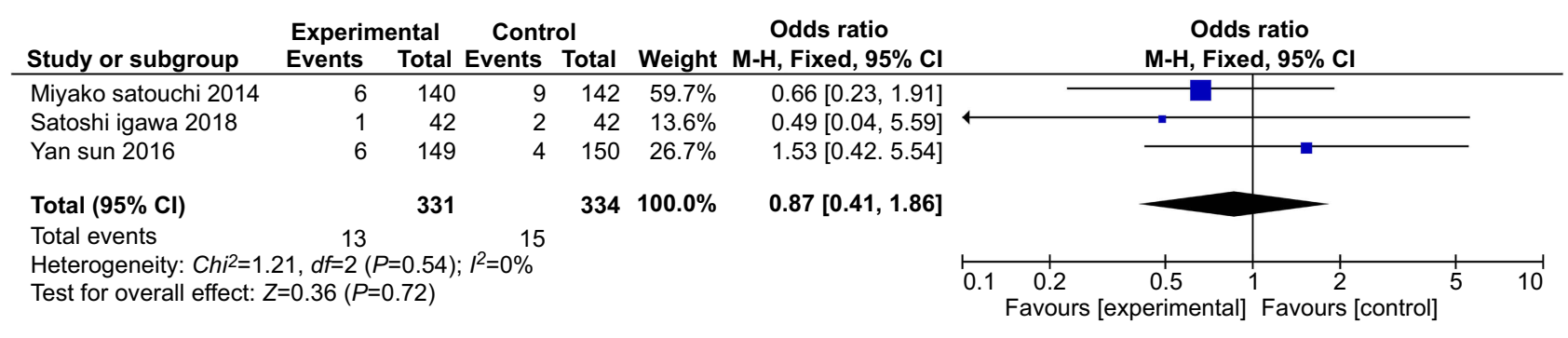

Figure 7 Pooled analysis of nausea comparing AMR versus chemotherapy.

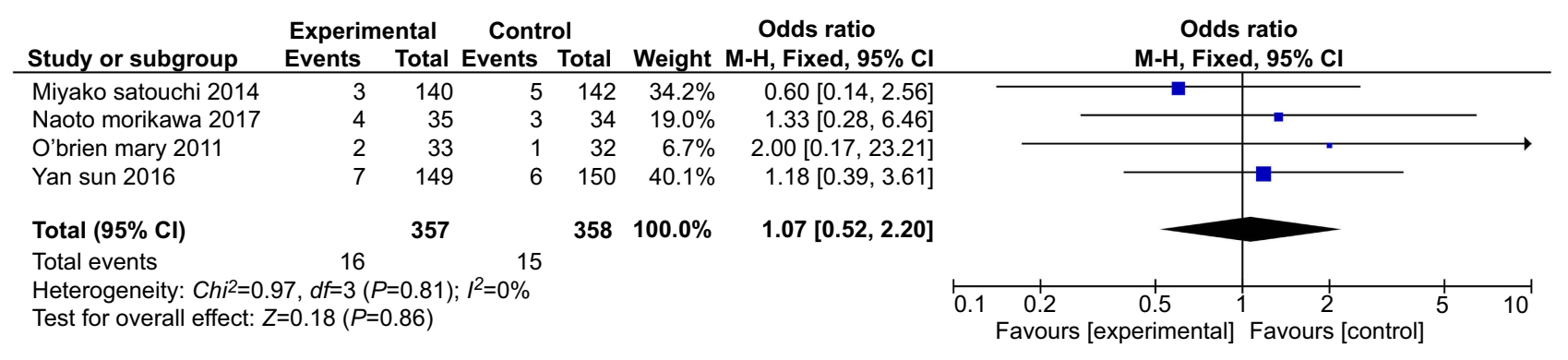

Figure 8 Pooled analysis of vomiting comparing AMR versus chemotherapy.

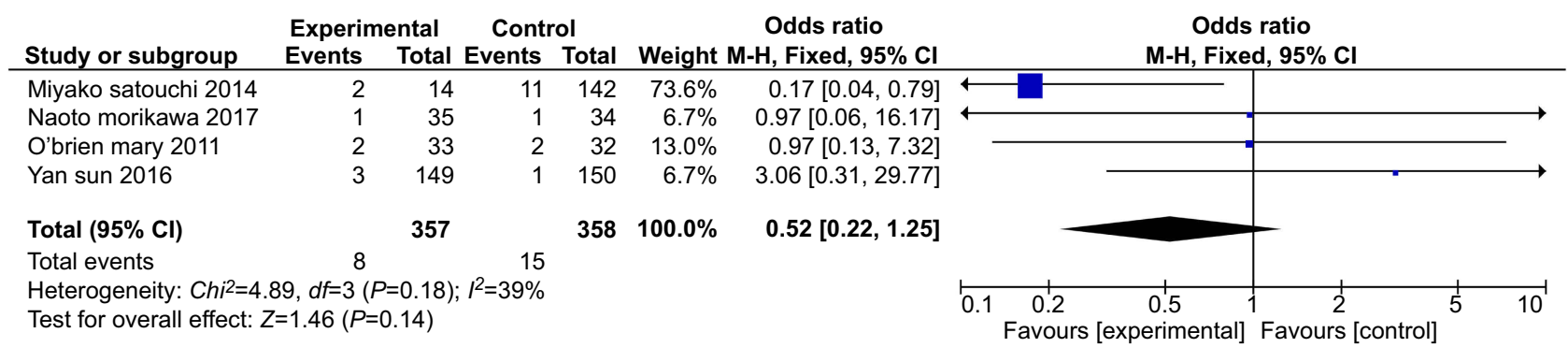

Figure 9 Pooled analysis of diarrhea comparing AMR versus chemotherapy.

Abbreviation: AMR, amrubicin.

In addition, AMR proved to be inferior to the control therapy, but the results seen here do not negate the effect of this agent for previously untreated SCLC and perhaps emphasize the particular value of AMR as later-line therapy in this setting. In terms of the safety, the main severe toxicity of amrubicin is myelosuppression, with neutropenia seen more frequently than thrombocytopenia or anemia. Careful hematological toxicity control is 


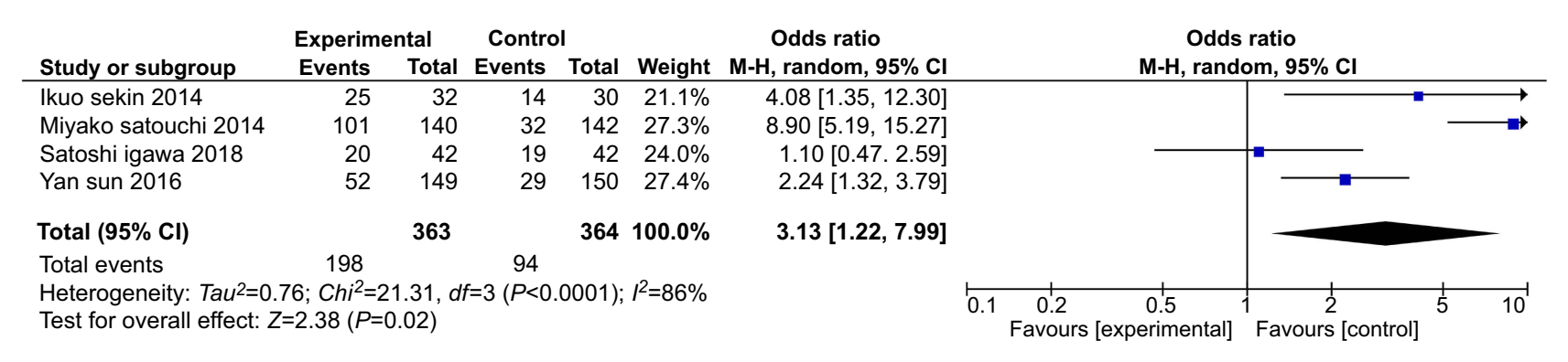

Figure 10 Pooled analysis of leukopenia comparing AMR versus chemotherapy. Abbreviation: AMR, amrubicin.

\begin{tabular}{|c|c|c|c|c|c|}
\hline \multirow[b]{2}{*}{ Study or subgroup } & \multicolumn{2}{|c|}{ Experimental } & \multicolumn{2}{|c|}{ Control } & \multirow[b]{2}{*}{ Weight } \\
\hline & Events & Total & Events & Total & \\
\hline Ikuo sekin 2014 & 29 & 32 & 24 & 30 & $13.1 \%$ \\
\hline Miyako satouchi 2014 & 134 & 140 & 83 & 142 & $17.6 \%$ \\
\hline Naoto morikawa 2017 & 31 & 35 & 18 & 34 & $14.9 \%$ \\
\hline O'brien mary 2011 & 24 & 33 & 22 & 32 & $16.2 \%$ \\
\hline Satoshi igawa 2018 & 27 & 42 & 17 & 42 & $17.7 \%$ \\
\hline Yan sun 2016 & 81 & 149 & 66 & 150 & $20.5 \%$ \\
\hline Total $(95 \% \mathrm{Cl})$ & & 431 & & 430 & $100.0 \%$ \\
\hline $\begin{array}{l}\text { Total events } \\
\text { Heterogeneity: } \operatorname{Tau}^{2}=0 \\
\text { Test for overall effect: }\end{array}$ & $\begin{array}{c}326 \\
C^{2}=2 \\
70(P=0\end{array}$ & 50, $d f=$ & $\begin{array}{r}230 \\
=5(P<0.0\end{array}$ & 017,1 & $=81 \%$ \\
\hline
\end{tabular}

Figure I I Pooled analysis of neutnpenia com paring AMR versus chemotherapy. Abbreviation: AMR, amrubicin.

essential with the use of amrubicin. The rate of grade 3 or worse neutropenia was $84.8-95.1 \%$ in previous studies, ${ }^{11,12}$ and the degree of myelosuppression and its risk of secondary serious infection and sepsis were containable with protocol-specific dose reductions, treatment delays, and g-CSF support and antibiotics.

However, some reports still reported sufficient efficacy compared amrubicin with approved drugs for the therapy of SCLC, even though the high incidence of toxicity. ${ }^{18}$ Its efficacy and alternate mechanism make it a potential candidate to treat this disease. More effective evidence for amrubicin to treat SCLC patients is warranted.

This study has several limitations that should be considered. First, due to small number of patients to draw any valid conclusions, bias exist, which may impact the results. Further investigations of this regimen in a large-scale study with greater statistical power are needed. Furthermore, though all included studies are all designed as random control trials (RCTs). However, heterogeneity due to varying experimental methods cannot be discounted entirely.

\section{Conclusion}

In summary, our meta-analysis indicates that AMR therapy demonstrates non-inferiority to the standard first-line
Odds ratio Odds ratio

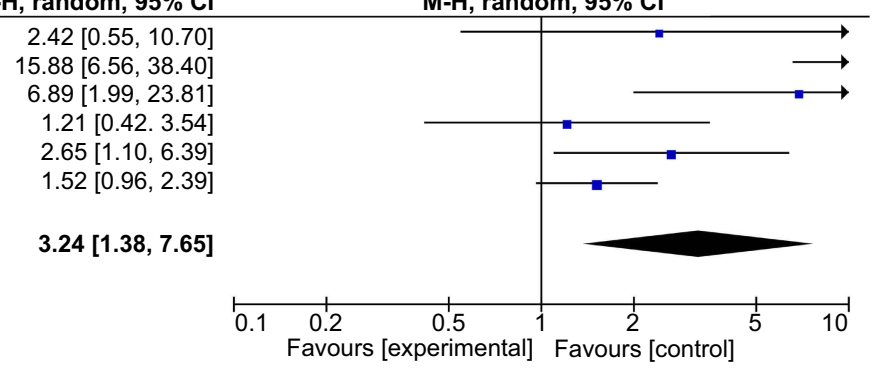

$2.42[0.55,10.70]$

5.88 $[6.56,38.40]$

$89[1.99,23.81]$

$65[1.10,6.39]$

$[0.96,2.39]$

Favours [experimental] Favours [control] chemotherapy with respect to survival, objective response, and safety in the treatment of previously untreated patients with ED-SCLC. Whether AMR regimen could be treated as a candidate for the first-line treatment of ED-SCLC still needs to be investigated.

\section{Acknowledgment}

No funding was received for this study.

\section{Disclosure}

The authors report no conflicts of interest in this work.

\section{References}

1. Siegel R, Naishadham D, Jemal A. Cancer statistics, 2012. CA Cancer J Clin. 2012;62(1):10-29. doi:10.3322/caac.20138

2. Tjanheijnen VCG. Diagnosis and treatment of lung cancer. An evidence-based guide for the practicing clinician. Mayo Clin Proc. 2001;76(9):962-963. doi:10.1016/S0025-6196(11)62123-X

3. Shepherd FA, Crowley J, Van Houtte P, et al. The international association for the study of lung cancer lung cancer staging project: proposals regarding the clinical staging of small cell lung cancer in the forthcoming (seventh) edition of the tumor, node, metastasis classification for lung cancer. $J$ Thoracic Oncol. 2007;2(12):1067-1077. doi:10.1097/JTO.0b013e31815bdc0d

4. Ferlay J, Parkin DM, Steliarova-Foucher E. Estimates of cancer incidence and mortality inEurope in 2008. Eur $J$ Cancer. 2010;46:765-781. doi:10.1016/j.ejca.2009.12.014 
5. Noda K, Nishiwaki Y, Kawahara M, et al. Irinotecan plus cisplatin compared with etoposide plus cisplatin for extensive small-cell lung cancer. N Engl J Med. 2002;346(2):85-91.

6. Satouchi M, Kotani Y, Shibata T, et al. Phase III study comparing amrubicin plus cisplatin with irinotecan plus cisplatin in the treatment of extensive-disease small-cell lung cancer: JCOG 0509. J Clin Oncol. 2014;32(12):1262-1268. doi:10.1200/JCO.2013.54.6911

7. Zatloukal P, Cardenal F, Szczesna A, et al. A multicenter international randomized phase III study comparing cisplatin in combination with irinotecan or etoposide in previously untreated small-cell lung cancer patients with extensive disease. Ann Oncol. 2010;21 (9):1810-1816. doi:10.1093/annonc/mdq036

8. Hermes A, Bergman B, Bremnes R, et al. Irinotecan plus carboplatin versus oral etoposide plus carboplatin in extensive small-cell lung cancer: a randomized phase III trial. J Clin Oncol. 2008;26 (26):4261-4267. doi:10.1200/JCO.2007.15.2777

9. Weikersthal LFV, Link H, Eberhardt WEE, et al. A German multicenter, randomized phase III trial comparing irinotecancarboplatin with etoposidecarboplatin as first-line therapy for extensive-disease small-cell lung cancer. Ann Oncol. 2011;22(8):1798-1804. doi:10.1093/annonc/mdq731

10. Jr LP, Chansky K, Shibata T, et al. Common arm comparative outcomes analysis of phase 3 trials of cisplatin + irinotecan versus cisplatin + etoposide in extensive stage small cell lung cancer: final patient-level results from Japan clinical oncology group 9511 and southwest oncology group 01. Cancer. 2010;116(24):5710-5715. doi:10.1002/cncr.25532

11. Yana T, Negoro S, Takada M, et al. Phase II study of amrubicin in previously untreated patients with extensive-disease small cell lung cancer: West Japan Thoracic Oncology Group (WJTOG) study. Invest New Drugs. 2007;25(3):253-258. doi:10.1007/s10637-006-9012-9

12. Ohe Y. Phase I-II study of amrubicin and cisplatin in previously untreated patients with extensive-stage small-cell lung cancer. Ann Oncol. 2005;16(3):430-436. doi:10.1093/annonc/mdi081

13. Higgins JP, Thompson SG. Quantifying heterogeneity in a meta-analysis. Stat Med. 2002;21:1539-1558. doi:10.1002/sim.1186
14. Higgins JP, Thompson SG, Deeks JJ, et al. Measuring inconsistency in meta-analyses. BMJ. 2003;327:557. doi:10.1136/bmj.327.7420.895

15. Satoshi I, Masayuki S, Takahiro O, et al. Comparison of carboplatin plus etoposide with amrubicin monotherapy for extensive-disease small cell lung cancer in the elderly and patients with poor performance status. Thoracic Cancer. 2018;9:967-973.

16. Morikawa N, Inoue A, Sugawara S, et al. Randomized phase II study of carboplatin plus irinotecan versus carboplatin plus amrubicin in patients with chemo-naïve extensive-stage small-cell lung cancer: North Japan Lung Cancer Study Group (NJLCG) 0901. Lung Cancer. 2017;111:38-42. doi:10.1016/j.lungcan.20 17.06.016

17. Sekine I, Okamoto H, Horai T, et al. A randomized phase III study of single-agent amrubicin vs. carboplatin/etoposide in elderly patients with extensive-disease small-cell lung cancer. Clin Lung Cancer. 2014;15(2):96-102. doi:10.1016/j.cllc.2013.11.006

18. Sun Y, Cheng Y, Hao X, et al. Randomized phase III trial of amrubicin/cisplatin versus etoposide/cisplatin as first-line treatment for extensive small-cell lung cancer. BMC Cancer. 2016;16(1):265. doi:10.1186/s12885-016-2301-6

19. Mary ER, O’Brien, Konopa K, Lorigan P, et al. Randomised phase II study of amrubicin as single agent or in combination with cisplatin versus cisplatin etoposide as first-line treatment in patients with extensive stage small cell lung cancer - EORTC 08062. Eur $J$ Cancer. 2011;47(15):0-2330.

20. Greenlee RT, Murray T, Bolden S, Wingo PA. Cancer statistics. $C A$ Cancer J Clin. 2000;50(2000):7-33. doi:10.3322/canjclin.50.1.7

21. Inoue $\mathrm{A}$, Ishimoto $\mathrm{O}$, Fukumoto $\mathrm{S}$, et al. $\mathrm{A}$ phase II study of amrubicin combined with carboplatin for elderly patients with small-cell lung cancer: North Japan lung cancer study group trial 0405. J Thoracic Oncol. 2006;1(6):551-555. doi:10.1097/01243894200607000-00009

22. Yamazaki K, Maemondo M, Suzuki T, et al. A Phase I study of amrubicin combined with carboplatin for elderly patients with small-cell lung cancer. $J$ Thoracic Oncol. 2006;1(6):551-555. doi:10.1097/01243894-200607000-00009
OncoTargets and Therapy

\section{Publish your work in this journal}

OncoTargets and Therapy is an international, peer-reviewed, open access journal focusing on the pathological basis of all cancers, potential targets for therapy and treatment protocols employed to improve the management of cancer patients. The journal also focuses on the impact of management programs and new therapeutic agents and protocols on patient perspectives such as quality of life adherence and satisfaction. The manuscript management system is completely online and includes a very quick and fair peer-review system, which is all easy to use. Visit http://www.dovepress.com/ testimonials.php to read real quotes from published authors. 